\title{
Hand Written Character Recognition Using Twelve Directional Feature Input and Neural Network
}

\author{
Dayashankar Singh \\ Lecturer, Deptt. of CSE \\ M.M.M. Engineering College \\ Gorakhpur (UP), India
}

\author{
Sanjay Kr. Singh \\ B.Tech-IV(CSE) \\ M.M.M. Engineering College \\ Gorakhpur (UP), India
}

\author{
Dr. (Mrs.) Maitreyee Dutta \\ Asst. Professor, Deptt. of CS \\ NITTTR \\ Chandigarh, India
}

\begin{abstract}
In this paper, we have applied a new feature extraction technique to calculate only twelve directional feature inputs depending upon the gradients. Features extracted from handwritten characters are directions of pixels with respect to their neighboring pixels. These inputs are given to a back propagation neural network with one hidden layer and one output layer. An analysis has been also carried out to compare the recognition accuracy, training time and classification time of newly developed feature extraction technique with some of the existing techniques. Experimental result shows that the new approach provides better results as compared to other techniques in terms of recognition accuracy, training time and classification time. The work carried out in this paper is able to recognize all type of handwritten characters even special characters in any language.
\end{abstract}

\section{Keywords:}

Neural Network, Feature Extraction Technique,, Recognition Accuracy, Backpropagation neural network.

\section{INTRODUCTION}

Handwritten character recognition is a very interesting field for researchers. Many feasible techniques have been developed in this field. Technique based on extracting various features of handwritten characters and providing these features as input to an Artificial Neural Network is used very often.

In this paper the features of directions of pixels of the characters with respect to their neighboring pixels are extracted and given as input to the neural network. A backpropagation feed-forward neural network is used to recognize the handwritten characters. After training the network with back-propagation learning algorithm, high recognition accuracy can be achieved. The direction has been divided into 12 regions with each region covering angle of 30 degree, hence direction value of any pixel may have only 12 values assigned from 1 to 12. This approach increases the information content and gives better recognition rate with reduced recognition time.

An overview of the paper is as follows: In section II, a Character modeling, Skeltionization \& Normalization are discussed. It also covers various feature extraction techniques for handwritten character recognition. In section III, a brief review of the MLP for character recognition is introduced; Backpropagation neural network and the training algorithms are also described. In Section IV, Experimental results and analysis are provided. Finally, the conclusion and future scope have been offered in Section V.

\section{SYSTEM DESIGN}

The main functional modules in Handwritten Character Recognition System are: Image acquisition module, Directional feature extraction module, and neural network module. The block diagram in Figure 1 shows the orientation of these modules.

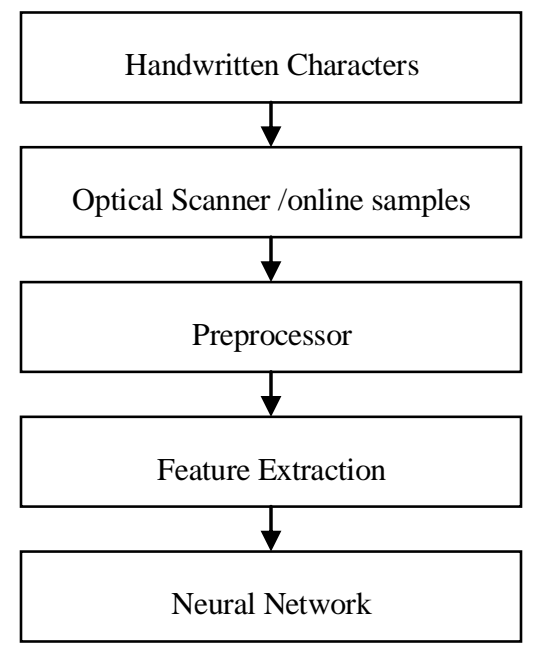

Figure 1 Block diagram of the recognition system

\section{A. Preprocessing}

- Noise removing

- Skeletonization

- Normalization

The function of this module is to remove noise by applying various digital image processing techniques and the image is normalized into $32 \times 32$ pixels. The output is a $32 \times 32$ pixels binary image of the character. Skeletonization refers to remove extra pixels and get only skeleton of character.

In this paper, mouse has been initialized in graphics mode and samples have been taken directly on the screen by various students and faculty members. A 32x 32 box has been created on screen and users are supposed to write in the box with the help of mouse. The samples taken on the screen are converted into three formats: 0 and 1 format, Gradients format and 12 
directional feature formats. These three formats have been stored in three different files and taken as input to the network.

\section{B. Feature Extraction}

Feature extraction is the process of collecting distinguishable information of an object or a group of objects so that on the basis of this information we can classify objects with different features. This is an important part of any recognition system.

\section{Conventional Feature Extraction}

In conventional method, if a line passes through a pixel, then corresponding pixel will be given value one (1), otherwise it is taken as zero (0). In this method the number of inputs are $32 \times 32=1024$ which requires more time to learn and classify the characters.

\section{Gradient Feature Extraction}

To find the gradients, we apply Sobel's mask to calculate the horizontal gradient (gx) and vertical gradient (gy) components as shown in Figure 2.

\begin{tabular}{|c|c|c|}
\hline 1 & 2 & 1 \\
\hline 0 & 0 & 0 \\
\hline-1 & -2 & -1 \\
\hline
\end{tabular}

\begin{tabular}{|l|l|l|}
\hline-1 & 0 & 1 \\
\hline-2 & 0 & 2 \\
\hline-1 & 0 & 1 \\
\hline
\end{tabular}

Horizontal component

Vertical component
Figure 2 Sobel's operator

We calculate the gradient of a pixel $(i, j)$ by using following formula.

$$
\begin{aligned}
& G x= g v(i, j)=f(i-1, j+1)+2 f(i, j+1)+f(i+1, j+1) \\
&-f(i-1, j-1)-2 f(i, j-1)-f(i+1, j-1) \ldots \ldots \ldots .(1) \\
& G y= g h(i, j)=f(i-1, j-1)+2 f(i-1, j)+f(i-1, j+1) \\
&-f(i+1, j-1)-2 f(i+1, j)-f(i+1, j+1) \ldots \ldots \ldots \ldots . . .(2) \\
& \quad \operatorname{grad}=\text { Gy/ Gx }=\tan -1[\operatorname{gh}(i, j) / g v(i, j)]
\end{aligned}
$$

In this way, the handwritten characters are converted into the gradient values ranging between 0 to 6.28 . It has been assumed that whenever a pixel is surrounded by all black pixels then its gradient values will be taken -1 .

\section{Directional Features}

After computing the gradient of each pixel of the character, we map these gradient values onto 12 direction values with angle span of 30 degree between any two adjacent direction values. The orientations of these 12 directional values are shown in Figure 3.

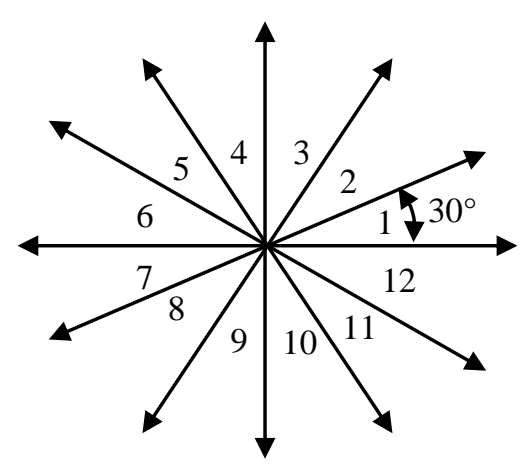

Figure 3 Orientation of directions

The mapping of gradient values on 12 directional values can be

\begin{tabular}{|c|c|c|c|}
\hline 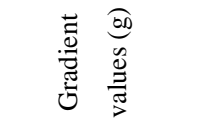 & 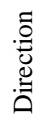 & 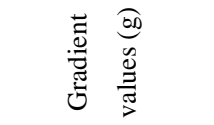 & 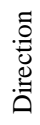 \\
\hline$g=-1$ & 0 & $\pi<\mathrm{g}<=7 \pi / 6$ & 7 \\
\hline $0<=g<=\pi / 6$ & 1 & $7 \pi / 6<\mathrm{g}<=4 \pi / 3$ & 8 \\
\hline$\pi / 6<\mathrm{g}<=\pi / 3$ & 2 & $4 \pi / 3<\mathrm{g}<=3 \pi / 2$ & 9 \\
\hline$\pi / 3<\mathrm{g}<=\pi / 2$ & 3 & $3 \pi / 2<\mathrm{g}<=5 \pi / 3$ & 10 \\
\hline$\pi / 2<\mathrm{g}<=2 \pi / 3$ & 4 & $5 \pi / 3<\mathrm{g}<=11 \pi / 6$ & 11 \\
\hline $2 \pi / 3<\mathrm{g}<=5 \pi / 6$ & 5 & $11 \pi / 6<\mathrm{g}<=2 \pi$ & 12 \\
\hline $5 \pi / 6<\mathrm{g}<=\pi$ & 6 & -- & -- \\
\hline
\end{tabular}
calculated by generalized formula as given below.

Direction $[\mathbf{n}(\mathbf{i}, \mathbf{j})]=\left(30(\mathbf{n}-1)^{\circ}\right) \leq \operatorname{grad}(\mathbf{i}, \mathbf{j})<\left(30 n^{\circ}\right)$

The detail values of 12 direction features can be shown in Figure 4.

Figure 4 Mapping of gradient on direction values

These 1024 direction values for $32 \times 32$ pixels are input to the neural network where each direction value is between 1 to 12 .If a pixel is surrounded by all the pixels having values zero then is its gradient assigned as 1. During the calculation of directional feature if gradient values are -1 then its directional feature values are assigned the values zeros(0s).

\section{BACKPROPAGATION NEURAL NETWORK}

A neural network is a network with nodes of processing elements, and connections as data carriers. The architecture of neural network determines how inputs of the neural network are transformed into an output. We have implemented a multi-layer feed forward neural network with one hidden layer as shown in Figure 5. 


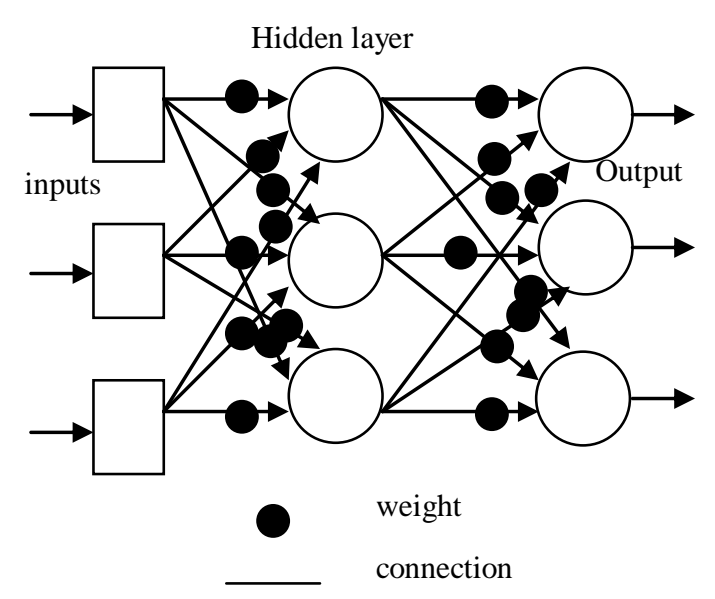

Figure 5 network model

The topology of the network is 1024 input nodes, 36 hidden nodes and 5 output nodes. Since character is normalized into $32 \times 32$ pixel images and corresponding to each pixel we have a directional value as input hence total number of inputs are 1024 to the neural network. The hidden layer has 32 neurons which are connected to all 1024 inputs. The output layer has 5 nodes since we have tested five types of handwritten characters: three Hindi characters, one English character and one special character.

Learning is implemented using the back-propagation algorithm with learning rate 0.2 . Gradient is calculated after every iteration and compared with threshold gradient value $10-10$. If gradient is greater than the threshold value then it performs next iteration. The batch steepest descent training function is traingd. The weights and biases are updated in the direction of the negative gradient of the performance function.

\section{EXPERIMENTAL RESULT}

Experiment has been carried out on 500 handwritten samples for training the network and 250 samples for testing the network. The samples include handwritten Hindi characters, English characters and some special characters. Both training and classification was performed on networks having 12 hidden layers respectively and results are shown in the Table 1.

\begin{tabular}{|c|c|c|c|c|c|c|c|}
\hline 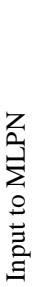 & 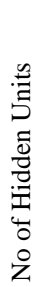 & 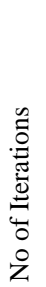 & 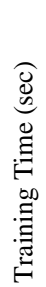 & 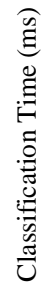 & 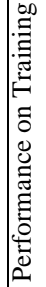 & 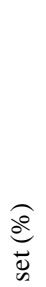 & 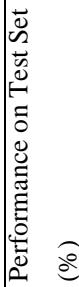 \\
\hline
\end{tabular}

\begin{tabular}{|c|c|c|c|c|c|c|}
\hline $\begin{array}{l}32 \times 32 \\
\text { Pixel input }\end{array}$ & 12 & 50 & 970 & 946 & 100 & 75 \\
\hline $\begin{array}{l}\text { 30x30 } \\
\text { Gradient } \\
\text { input }\end{array}$ & 12 & 50 & 764 & 246 & 100 & 90 \\
\hline $\begin{array}{l}30 \times 30, \\
\text { Twelve } \\
\text { Directional } \\
\text { input }\end{array}$ & 12 & 50 & 421 & 221 & 100 & 97 \\
\hline
\end{tabular}

\section{Table 1 Result Analysis}

Experiment shows that pixel input feature extraction technique is giving poor accuracy and it also requires more time to train the network. Experiment also shows that this technique requires more number of iterations for improving the accuracy. It has been also shown that it requires more classification time. Experimental result shows that the 12 directional feature extraction techniques provides better result in terms of Recognition accuracy, Training time and Classification time. In 12 directional feature extraction technique, experiment has been carried out on discrete integer, a value which provides more information content due to which learning becomes easy and training requires less time. The recognition accuracy is high only because of having more information content in 12 direction feature extraction technique.

\section{CONCLUSION \& FUTURE SCOPE}

In this work a lot of efforts have been made towards increasing the accuracy of handwritten character recognition. In this work, 12 directional input feature extraction technique has been introduced, which provides high accuracy and less training time for handwritten character recognition. This work can be further extended to the character recognition of other languages as well as for special character recognition. For doing so, sufficient training and also a sufficient number of samples are required so that network could be trained for them. Although, a lot of efforts have been made to complete a great deal of work but still it has tremendous scope for further improvement/ enhancement. In future, efforts can be made to improve the recognition accuracy of the network for special characters by using more training samples and by making advancements in 12 directional feature extraction systems. An automatic system for recognizing all handwritten characters can also be developed. A brief outline about the future scope of this work can be given as follows.

Scanned samples can be taken as input to the network and it is expected that there will be $100 \%$ accuracy in 12 directional input Feature Extraction Technique (A new approach). This work can be extended for word recognition in any language by including regular expressions. It can also be extended for language translation by including regular expressions and grammar. 


\section{REFFERENCES}

1. Cheng-Lin Liu, "Normalization-Cooperated Gradient Feature Extraction for Handwritten Character Recognition" IEEE Transactions on Pattern Analysis and Machine Intelligence, Volume 29, Issue 8, Aug. 2007 Page(s) 1465-1469.

2. Hailong Liu, Xiaoqing Ding, "Handwritten character recognition using gradient feature and quadratic classifier with multiple discrimination schemes" Eighth IEEE International Conference on Document Analysis and Recognition, Vol. 1, Page(s): 19-23, August 29-1 Sept., 2005.

3. K. Yamada and H. Kami, "Handwritten numeral recognition by multilayered neural network with improved learning algorithm," IJCNN Washington DC, vol. 2, pp. 259-266, 1989.

4. S.J. Smith and M.O. Baurgoin, "Handwritten character classification using nearest neighbor in large database," IEEE Trans. on Pattern and Machine Intelligence, vol. 16, pp. 915-919, Oct. 1994.
5. Starzyk,J.A.Ansari, “ Feedforward neural network for handwritten character recognition", IEEE International Symposium on Circuits and Systems(ISCAS), Volume 6, Page(s) 2884-2887, 1992.

6. Sutha.J, Ramraj.N, "Neural Network Based Offline Tamil Handwritten Character Recognition System", IEEE International Conference on Computational Intelligence and Multimedia Application, 2007 Volume 2, 13-15, Dec.2007, Page(s): 446-450, 2007.

7. Verma B.K, "Handwritten Hindi Character Recognition Using Multilayer Perceptron and Radial Basis Function Neural Network", IEEE International Conference on Neural Network, vol.4, pp. 2111-2115, 1995. 